\title{
Collapsing the list of myocardial infarction-related differentially expressed genes into a diagnostic signature
}

\author{
German Osmak $^{1^{*}}$ (D), Natalia Baulina ${ }^{2}$, Philipp Koshkin ${ }^{1}$ and Olga Favorova ${ }^{2}$
}

\begin{abstract}
Background: Myocardial infarction (MI) is one of the most severe manifestations of coronary artery disease (CAD) and the leading cause of death from non-infectious diseases worldwide. It is known that the central component of CAD pathogenesis is a chronic vascular inflammation. However, the mechanisms underlying the changes that occur in T, B and NK lymphocytes, monocytes and other immune cells during CAD and MI are still poorly understood. One of those pathogenic mechanisms might be the dysregulation of intracellular signaling pathways in the immune cells.

Methods: In the present study we performed a transcriptome profiling in peripheral blood mononuclear cells of Ml patients and controls. The machine learning algorithm was then used to search for Ml-associated signatures, that could reflect the dysregulation of intracellular signaling pathways.

Results: The genes ADAP2, KLRC1, MIR21, PDGFD and CD14 were identified as the most important signatures for the classification model with L1-norm penalty function. The classifier output quality was equal to 0.911 by Receiver Operating Characteristic metric on test data. These results were validated on two independent open GEO datasets. Identified Ml-associated signatures can be further assisted in MI diagnosis and/or prognosis.

Conclusions: Thus, our study presents a pipeline for collapsing the list of differential expressed genes, identified by high-throughput techniques, in order to define disease-associated diagnostic signatures.
\end{abstract}

Keywords: Transcriptomics, Machine learning, Myocardial infarction, miRNA, Transcriptional signatures

\section{Background}

Myocardial infarction (MI) is one of the most severe manifestations of coronary artery disease (CAD) and the leading cause of death from non-infectious diseases worldwide [1]. In most cases, MI occurs as a serious complication of atherosclerosis-a complex disease, the etiology of which is still not fully elucidated [2]. Recent studies have shown that the central component of atherosclerosis pathogenesis is a chronic vascular inflammation, resulting in endothelial dysfunction and, consequently, in

\footnotetext{
*Correspondence: german.osmak@gmail.com

1 National Medical Research Center for Cardiology, Moscow 121552, Russia

Full list of author information is available at the end of the article
}

an increased probability of hemodynamic abnormalities, including through the thrombosis [3]. Such a vascular lesion is emerged with the leading involvement of intimal cells (fibroblasts, endothelial and smooth muscle cells) and peripheral blood mononuclear cells (PBMC) [4]. The immune system cells are also actively involved in the processes accompanying MI; initiating aseptic inflammation, they contribute to the removal of cell debris from the injured area, remodeling and repair of heart tissue [5, 6]. The area of myocardial injury and the disease progression depend on the activity of different processes, including on the inflammatory phase in the first days after MI [5]. However, the pathogenic mechanisms underlying the changes that occur in PBMC (T, B and NK lymphocytes and monocytes) during atherosclerosis and MI are still

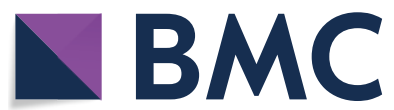

(c) The Author(s) 2020. This article is licensed under a Creative Commons Attribution 4.0 International License, which permits use, sharing, adaptation, distribution and reproduction in any medium or format, as long as you give appropriate credit to the original author(s) and the source, provide a link to the Creative Commons licence, and indicate if changes were made. The images or other third party material in this article are included in the article's Creative Commons licence, unless indicated otherwise in a credit line to the material. If material is not included in the article's Creative Commons licence and your intended use is not permitted by statutory regulation or exceeds the permitted use, you will need to obtain permission directly from the copyright holder. To view a copy of this licence, visit http://creativeco mmons.org/licenses/by/4.0/. The Creative Commons Public Domain Dedication waiver (http://creativecommons.org/publicdomain/ zero/1.0/) applies to the data made available in this article, unless otherwise stated in a credit line to the data. 
poorly understood. One of those mechanisms might be the dysregulation of intracellular signaling pathways in immune cells [7].

One way to establish MI transcriptional signatures, which include both dysregulated individual genes and signaling pathways, containing dysregulated genes, is to simultaneously study of transcriptional profiles of protein-coding genes and genes for regulatory non-coding RNA. Among non-coding RNAs, miRNAs are of particular interest in the context of robustness of biological processes, since they regulate key elements of extensive segments of signaling pathways' networks [8-10]. To date, consistency of expressional changes of miRNAs and their target genes has been investigated in macrophages of pigs and rats with experimental MI [11] and in the whole blood of MI patients [12].

In the present study, we performed a transcriptome profiling in PBMC in MI patients 24-36 h after disease onset and healthy individuals and revealed MI-associated signatures, consisting of individual protein-coding genes or functional patterns of genes, such as miRNA with its co-expressed target genes or combination of co-expressed genes, attributed to a definite signaling pathway.

\section{Materials and methods}

\section{Pipeline}

The pipeline of the study design is illustrated in Fig. 1. RNA Microarray analysis was used to identify genes that were significantly $(p<0.05)$ associated with MI (differentially expressed genes, DEGs). Those DEGs that have passed threshold for multiple comparisons were considered MI transcriptional signatures. Functional patterns of co-expressed DEGs were also considered MI transcriptional signatures. Such functional patterns included i) differentially expressed miRNA and its coexpressed target mRNA(s) and ii) DEGs attributed to a Reactome gene set. In the latter case, the Reactome gene sets were considered the most informative if they (i) account for more than $10 \%$ of all co-expressed DEGs and/or (ii) include DEGs passed multiple comparisons correction.

The validation of identified MI transcriptional signatures was performed on two open data sets; DEGs which were not validated on at least one of these sets were excluded from further consideration. The DEGs within validated MI transcriptional signatures were used to construct binary classifiers. Given the high quality of classification and stability of the detected composite transcriptional biomarker, a logistic regression with the L1-norm penalty function was used to select the most significant DEGs on test dataset.

\section{Patients and controls}

Six patients (all men, mean age $51.3 \pm 5.9$ years) with first ST-segment elevation MI were enrolled in this study. All patients were diagnosed at the National Medical Scientific Center for Cardiology (Moscow, Russia) based on symptoms of myocardial ischemia, increase of high-sensitivity cardiac troponin I (hs-cTn-I) and/or

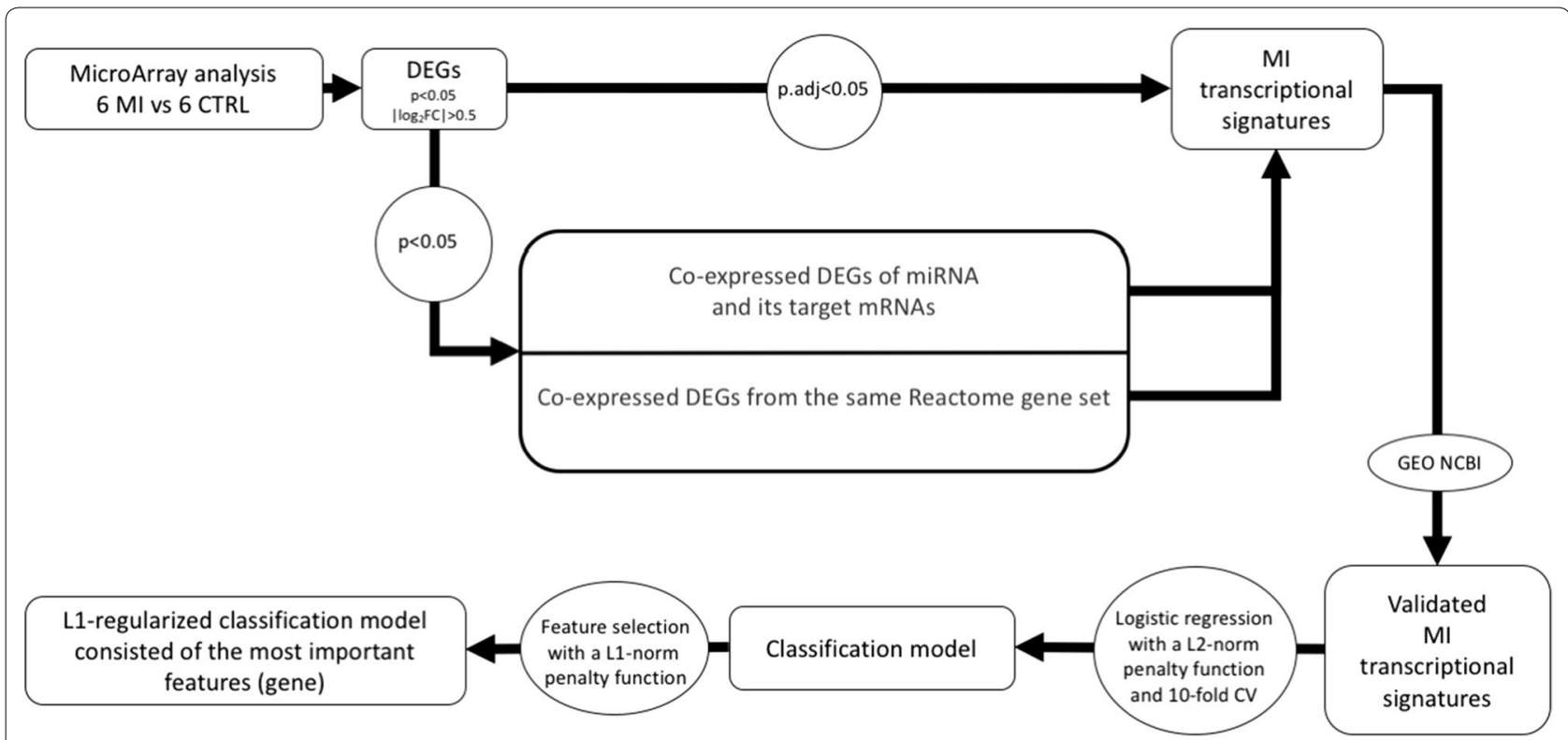

Fig. 1 A schematic pipeline of the study for MI transcriptional signatures' identification. DEGs differentially expressed genes. M/ patients with myocardial infarction. CTRLs individuals in the control group, CV cross-validation 
emergence of new or presumed new ST-segment elevation, new left bundle branch block or development of pathological Q waves in accordance with Third Universal Definition of MI [13]. Hs-cTn-I was measured during the initial patient assessment (from 1 to $18 \mathrm{~h}$ after the onset of disease symptoms). All patients underwent coronary angiography on admission and were treated according to contemporary guidelines. The characteristics of MI patients are presented in Table 1. A total of 6 individuals of the control group (CTRLs) (all men, mean age $51.0 \pm 7.1$ years) with normal electrocardiogram, no history of CVD and diabetes mellitus were included in the study; CTRLs characteristics by smoking status, age and body mass index were compatible to MI patients. All participants lived in European Russia. The ethical approval was obtained from the local Ethics Committee, and written informed consent had been received from each person in accordance with the Declaration of Helsinki.

\section{Peripheral blood mononuclear cells collection and RNA extraction}

Blood samples were collected in the morning from MI patients (24-36 h after the disease onset) and CTRLs. PBMC were isolated using Ficoll-Hypaque density gradient method (Sigma-Aldrich, St. Louis, MO, USA) within $3 \mathrm{~h}$ of sampling. Total RNA including small RNA was extracted using miRNeasy Mini Kit (Qiagen, Hildren, Germany) following the manufacturer's instructions. The RNA quantity was measured using the NanoDropTM spectrophotometer (Thermo Fisher Scientific, Waltham,

\section{Table 1 Baseline characteristics of MI patients (all men)}

\begin{tabular}{|c|c|}
\hline Total number & 6 \\
\hline Mean age $\pm S D$ (years) & $51.3 \pm 5.9$ \\
\hline Body mass index $\pm S D,\left(\mathrm{~kg} / \mathrm{m}^{2}\right)$ & $28.2 \pm 3.3$ \\
\hline Smoking (\%) & 68 \\
\hline Total cholesterol \pm SD, $(\mathrm{mmol} / \mathrm{l})$ & $5.2 \pm 1.3$ \\
\hline Triglycerides $\pm S D,(\mathrm{mmol} / \mathrm{l})$ & $1.4 \pm 0.34$ \\
\hline LDL cholesterol \pm SD, $(\mathrm{mmol} / \mathrm{l})$ & $2.6 \pm 1.4$ \\
\hline HDL cholesterol \pm SD, (mmol/l) & $1.0 \pm 0.1$ \\
\hline $\begin{array}{l}\text { Left ventricular ejection fraction at the time of Ml onset } \\
(\%)\end{array}$ & $55.2 \pm 6.0$ \\
\hline High-sensitivity cardiac troponin I (hs-cTn-l) \pm SD, (pg/ml) & $1857.9 \pm 98.5$ \\
\hline Previous MI (n) & 0 \\
\hline Previous revascularization (n) & 0 \\
\hline Coronary obstructive/non-obstructive MI (n) & $3 / 3$ \\
\hline Hypercholesterolemia (n) & 2 \\
\hline \multicolumn{2}{|l|}{ Accompanied diseases } \\
\hline Diabetes mellitus before MI ( $n$ ) & 0 \\
\hline Essential hypertension before MI (n) & 3 \\
\hline Chronical bronchitis (n) & 2 \\
\hline
\end{tabular}

MA, USA); the RNA integrity was assessed by QIAxcel Advanced System (Qiagen, Hilden, Germany). Samples with RNA integrity number (RIN) value above eight were included in subsequent experiments.

\section{RNA microarray analysis}

The transcriptome analysis was performed using GeneChip Human Transcriptome Array 2.0, which provides the ability to analyze the expression of 44,699 proteincoding genes and 22,829 non-protein coding genes, including 1346 miRNA genes (ThermoFisher Scientific, Santa Clara, CA, USA). Briefly, total RNA (500 ng) of samples were each proceeded to poly(A)tailing and biotin ligation reactions using FlashTag Biotin HSR RNA Labeling Kit (ThermoFisher Scientific, Santa Clara, CA, USA). The biotin-labeled RNA samples were hybridized on GeneChip Human Transcriptome Array 2.0 using manufacturer's instructions and scanned on the GeneChip Scanner 7G System. Computational analysis of the microarray data files was performed using $\mathrm{R}$ programming language version 3.5.1. Data processing was carried out based on the affy package written in R [14]. A biomaRt package was used to annotate the obtained data [15]. Probes demonstrating evidence for cross-hybridization, i.e. transcript sequences annotated to more than two coding genes were excluded from this study. If transcripts belong to the same gene ID, a transcript with the most detectable expression level was selected. To detect differentially expressed genes, calculate the levels of statistical significance and adjust them for multiple comparisons by Benjamini-Hochberg procedure ( $p$ and $p_{\text {adj }}$, respectively) the standard limma package protocol was used [16]. All expression data are deposited in the Gene Expression Omnibus international public repository under accession identification as GSE141512 [17].

\section{Bioinformatic analysis}

MirTarBase was used to select experimentally validated target genes for miRNAs [18]. Gene set enrichment analysis (over-representation analysis) was performed using Tools of Reactome Database [19].

To construct and analyze the gene-gene interaction networks, NetworkX 2.0 package for Python was used [20]. STRING database [21] was used to find proteinprotein interactions.

\section{Statistics analysis and machine learning}

Statistical analysis was performed using $\mathrm{R}$ programming language version 3.5.1. Null hypotheses were rejecting if $\mathrm{p}<0.05$. To study the dependence/correlation of two continuous random variables, the Spearman's Rank Correlation test was used. The logistic regression classifier was trained using the tools of scikit-learn v0.20.3 for 
Python [22]. To reduce the possible classification model overfitting the 12-norm regularization and tenfold cross validation were used. The selection of the optimal regularization coefficient was performed by grid search using the GridSearchCV () function. The quality of the classification model was estimated by the areas under receiver operating characteristic curve (ROC-AUC). The final assessment of the quality of the classification model was carried out on the test dataset that was not used for training. For training and testing the model z-scaling of continuous features was performed at the preprocessing data stage.

\section{Validation analysis}

The Gene Expression Omnibus database (GEO, http:// www.ncbi.nlm.nih.gov/geo) was used in order to validate the obtained results. Two open data sets-GSE59867 and GSE62646 with gene expression profiles in PBMC of MI patients and healthy individuals without a history of CVD were investigated; they were obtained on GeneChip Human Gene 1.0 ST Array [transcript (gene) version]. The dataset GSE59867 included expression data of 111 MI cases and 48 CTRLs; the dataset GSE62646-of $28 \mathrm{MI}$ patients and 14 CTRLs (mixed-gender sets).

\section{Results}

\section{Array-based transcriptome profiling}

Transcriptome profiling in PBMC of six MI patients and six gender- and age-matched control individuals (CTRLs) was performed using GeneChip Human Transcriptome Array 2.0 (Fig. 1). As a result, a total of 84 differentially expressed genes (DEGs) were identified $\left(-0.5<\log _{2} \mathrm{FC}>0.5, p<0.05\right)$ (Additional file 1: Table S1), from which 48 protein-coding genes and 2 miRNA genes (MIR21 and MIR223) were upregulated, while 34 proteincoding genes were downregulated in MI patients. Among all observed DEGs KLRB1 and ADAP2 passed the threshold for multiple comparisons correction $\left(\log _{2} \mathrm{FC}=-0.64\right.$, p.adj $=0.0454$ and $\log _{2} \mathrm{FC}=0.64$, p.adj $=0.0495$, respectively); both these genes were further considered as MI transcriptional signatures.

\section{The search for MI transcriptional signatures: miRNA and its target mRNA(s)}

Among identified DEGs $(p<0.05)$ presented in Fig. 2, BCL6, CCR1, PDGFD, SGK1, and TGFBR3 genes were found to be targets of miR-21, while MAFB-target of miR-223 based on MirTarBase database. As assessed by Spearman's correlation analysis the expression levels of $B C L 6, C C R 1$, and $S G K 1$ were positively correlated $(p<0.001$, Ro $>0.9)$ and PDGFD and TGFBR3 -negatively correlated $(p<0.05$; Ro $<-0.6)$ with MIR21 expression level in MI patients and CTRLs (Additional file 1:

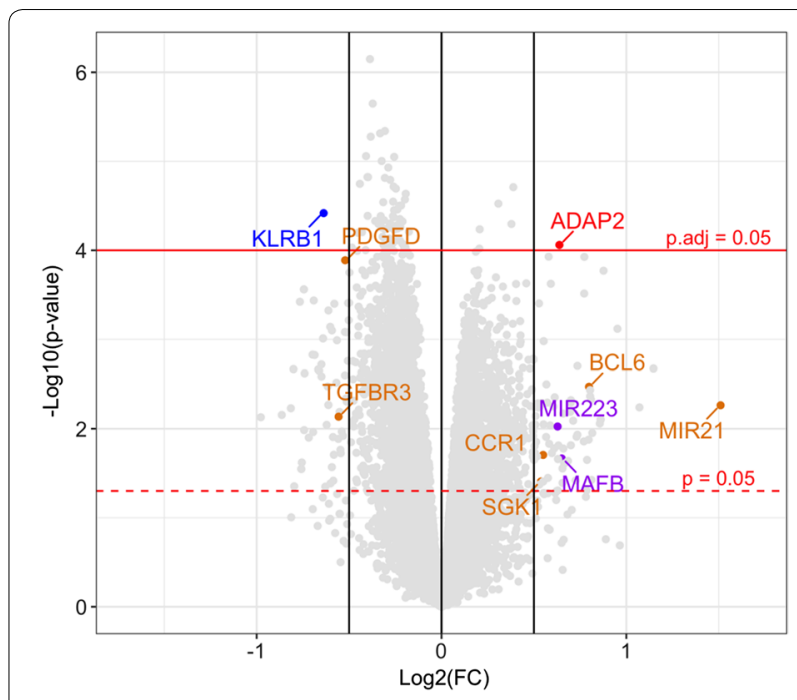

Fig. 2 Volcano plot of gene expression changes in PBMC of MI patients compared to CTRLs. Blue dot indicates downregulated gene $\left(\log _{2} \mathrm{FC}<-0.5\right)$; red dot indicates upregulated gene $\left(\log _{2} \mathrm{FC}>0.5\right)$, which passed threshold for multiple comparisons ( $p$.adj $<0.05$ ); Among differentially expressed genes (DEGs) MIR21 and its target genes are marked in orange, MIR223 and its target gene - in purple $\left(-0.5<\log _{2} \mathrm{FC}>0.5, p<0.05\right)$

Figure S1). A positive correlation between the expression levels of MAFB and MIR223 $(p<0.1, \mathrm{Ro}=0.5)$ was also observed (Additional file 1: Figure S2). Thus, MIR21 and MIR223 genes, together with their functionally associated co-expressed target genes, were considered as two MI transcriptional signatures.

\section{The search for MI transcriptional signatures: Reactome gene sets}

The enrichment analysis was undertaken in order to search for the functional patterns which included DEGs attributed to a Reactome gene set (Table 2). Nine Reactome gene sets were significantly overrepresented $(\mathrm{FDR}<0.05)$ among the 48 upregulated protein-coding genes (see above). The first three sets included each more than $10 \%$ of upregulated genes: "Immune system" -22 DEGs from 2663 genes presented in the set $(F D R=0.023)$, "Neutrophil degranulation" - 13 DEGs from 480 genes $(F D R=0.0035)$ and "Cytokine Signaling in Immune system" - 9 DEGs from 1055 genes $(F D R=0.015)$. "Immune system" gene set is at the highest level of the Reactome hierarchy and includes "Neutrophil degranulation" and "Cytokine Signaling in Immune system" pathways that are separately characterized by more significant overrepresentation of DEGs. So that, the DEGs from these two pathways were chosen to further analysis in the context of potential MI transcriptional signatures. Notably, "Cytokine 
Table 2 Reactome gene sets significantly overrepresented among the differentially expressed genes in PBMC from MI patients when compared to controls

\begin{tabular}{|c|c|c|c|c|c|}
\hline No & Reactome set name & $\begin{array}{l}\text { Total number } \\
\text { of genes } \\
\text { in the set }\end{array}$ & $\begin{array}{l}\text { Number of differentially } \\
\text { expressed genes (DEGs) }\end{array}$ & DEGs & FDR \\
\hline \multicolumn{6}{|c|}{ Upregulated genes } \\
\hline 1 & Immune system & 2663 & 22 & $\begin{array}{l}\text { BCL6, BST1, C3AR1, CCR1, CD14, CLEC4D, } \\
\text { CLEC6A, CR1, FCGR1A, FCGR1B, FLT3, } \\
\text { FPR1, FPR2, GRN, HLA-DQB1, PADI2, PYGL, } \\
\text { S100A12, SLC11A1, SOCS3, TLR2, TLR8 }\end{array}$ & 0.023 \\
\hline 2 & Neutrophil degranulation & 480 & 13 & $\begin{array}{l}\text { BST1, C3AR1, CD14, CLEC4D, CR1, FPR1, FPR2, } \\
\text { GRN, PADI2, PYGL, S100A12, SLC11A1, TLR2 }\end{array}$ & 0.0035 \\
\hline 3 & Cytokine Signaling in immune system & 1055 & 9 & $\begin{array}{l}\text { BCL6, CCR1, FCGR1A, FCGR1B, FLT3, FPR1, } \\
\text { HLA-DQB1, S100A12, SOCS3 }\end{array}$ & 0.015 \\
\hline 4 & Interferon gamma signaling & 250 & 4 & FCGR1A, FCGR1B, HLA-DQB1, SOCS3 & 0.015 \\
\hline 5 & Signaling by non-receptor tyrosine kinases & 70 & 2 & NRG1, SOCS3 & 0.033 \\
\hline 6 & Signaling by PTK6 & 70 & 2 & NRG1, SOCS3 & 0.033 \\
\hline 7 & PTK6 Activates STAT3 & 7 & 1 & SOCS3 & 0.033 \\
\hline 8 & GRB7 events in ERBB2 signaling & 6 & 1 & $N R G 1$ & 0.031 \\
\hline 9 & $\begin{array}{l}\text { Transport of glycerol from adipocytes to } \\
\text { the liver by Aquaporins }\end{array}$ & 3 & 1 & $A Q P 9$ & 0.015 \\
\hline \multicolumn{6}{|c|}{ Downregulated genes } \\
\hline 1 & $\begin{array}{l}\text { Immunoregulatory interactions between a } \\
\text { Lymphoid and a non-Lymphoid cell }\end{array}$ & 297 & 6 & $\begin{array}{l}K L R B 1, K L R C 1, K L R D 1, K L R F 1, K I R 2 D L 1, \\
\text { KIR2DL3 }\end{array}$ & 0.021 \\
\hline 2 & DAP12 signaling & 29 & 4 & $K L R C 1, K L R C 2, K L R C 3, K L R D 1$ & 0.021 \\
\hline
\end{tabular}

Signaling in Immune system" pathway involves BCL6 and CCR1-the target genes of miR-21, which were already included in one of the identified MI transcriptional signatures.

As can be seen from Table 2, two Reactome gene sets were significantly overrepresented among the 34 downregulated in MI genes: "Immunoregulatory interactions between a Lymphoid and a non-Lymphoid cell" (6 DEGs from 297 genes, FDR =0.021) and "DAP12 signaling" (4 DEGs from 29 genes, FDR $=0.021)$. Each of these sets includes more than $10 \%$ of the downregulated genes and is involved in signal transduction in lymphoid cells, namely in natural killers (NK). The "DAP12 signaling" pathway was overrepresented exclusively among the genes which encode the killer cell lectin-like receptors (KLR) expressed in NK cells, two of these DEGs (KLRD1 and $K L R C 1$ ) were as well observed in "Immunoregulatory interactions between a Lymphoid and a non-Lymphoid cell" pathway. The last mentioned pathway was also overrepresented by KIR $2 D L 1$ and KIR $2 D L 3$ genes, encoding a killer cell immunoglobulin-like receptors, the transmembrane glycoproteins expressed by NK and T cells' subsets. Notably, KLRB1 gene, defined previously individually as $\mathrm{MI}$ transcriptional signature $\left(\log _{2} \mathrm{FC}=-0.64\right.$, p.adj $=0.0454$ ) was included only in "Immunoregulatory interactions between a Lymphoid and a non-Lymphoid cell" pathway. The DEGs from this pathway were chosen for further analysis in the context of potential MI transcriptional signatures.

The search for interacting genes/proteins among the DEGs from selected Reactome sets "Neutrophil degranulation", "Cytokine Signaling in Immune system" and "Immunoregulatory interactions between a Lymphoid and a non-Lymphoid cell" (lines 2, 3 and 10 in Table 2) was performed using String database. Almost all the DEGs from the "Neutrophil degranulation" set (with the exception of the PADI2, GRN and PYGL genes) were found to interact among themselves (Fig. 3a). The expression levels of these 10 interacting genes were significantly positively correlated between each other $(0.93>$ Ro $>0.51$, $p<0.05$ ) (Additional file 1: Figure S3). Thus, we considered the pattern of interacting genes from this pathway, namely BST1, C3AR1, CD14, CLEC4D, CR1, FPR1, FPR2, $S 100 A 12, S L C 11 A 1$ and TLR2 as potential MI transcriptional signature.

In the "Cytokine Signaling in Immune system" gene set six interacting genes (CCR1, FCGR1A, FCGR1B, FPR1, $H L A-D Q B 1$ and $S 100 A 12$ ) were found (Fig. 3b), and the expression levels of these genes, with the exception of the $H L A-D Q B 1$, were positively correlated with each other $(0.99>\operatorname{Ro}>0.62, p<0.05)$ (Additional file 1: Figure S4). As previously mentioned, CCR 1 is the target gene of miR21, and has already been included to MIR21-containing MI transcriptional signature. According to correlation 

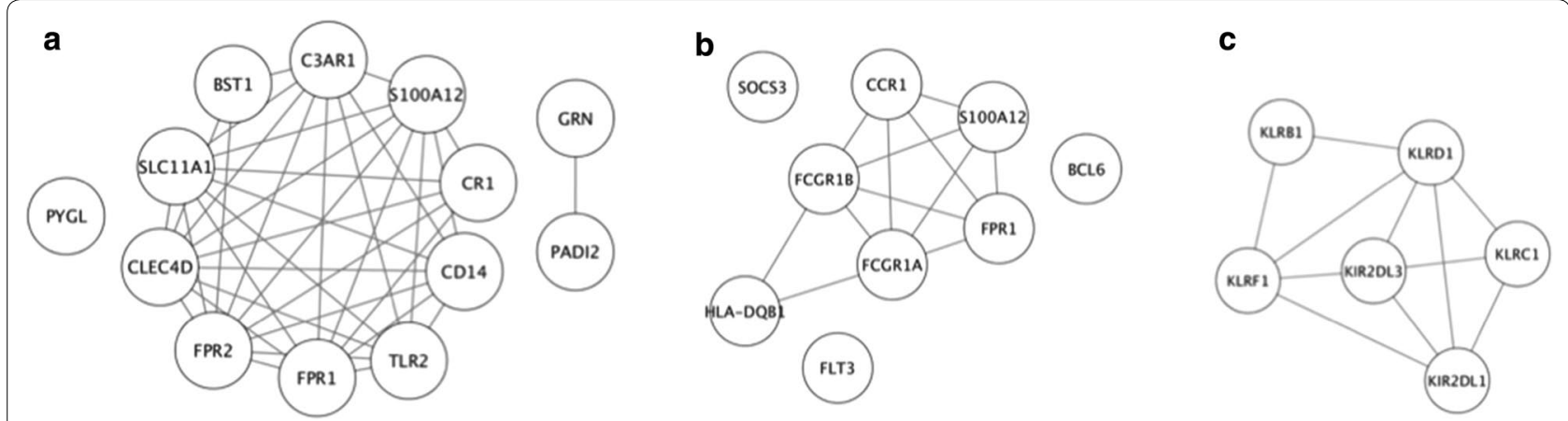

Fig. 3 Network analysis of the Reactome gene sets "Neutrophil degranulation" (a), "Cytokine Signaling in Immune system" (b) and

"Immunoregulatory interactions between a Lymphoid and a non-Lymphoid cell" (c). The edges indicate molecular interactions between nodes based on String database

analysis, $M I R 21$ expression level positively correlates not only with CCR1, but also with FCGR1A, FCGR1B, FPR1 and $S 100 A 12$ expression levels $(0.93>$ Ro $>0.71, p<0.01)$ (Additional file 1: Figure S4). Thus, we considered these genes as components that extend the MIR21-containing MI transcriptional signature.

In Reactome pathway "Immunoregulatory interactions between a Lymphoid and a non-Lymphoid cell" different interacting gene pairs were found between all the genes from this set: KLRB1, KLRC1, KLRD1, KLRF1, KIR2DL1, $K I R 2 D L 3$ (Fig. 3c) and they were predominantly characterized by significantly positive correlation between their expression levels $(0.88>\operatorname{Ro}>0.55, p<0.05)$ (Additional file 1: Figure S5). Noteworthy, KLRB1 gene whose differential expression passed correction for multiple comparisons and could be considered as the MI transcriptional signature, interacts with KLRD1 and KLRF1 from this gene set. Therefore, we included genes $K L R C 1, K L R D 1$, KLRF1, KIR2DL1 and KIR2DL3 in the KLRB1-containing MI transcriptional signature.

Overall, the conducted analysis allowed us identifying the following five $\mathrm{MI}$ transcriptional signatures containing all in all 29 DEGs: $\{A D A P\},\{K L R B 1+K L R C 1, K L R D 1$, KLRF1, KIR2DL1, and KIR2DL3\}, \{MIR21 + BCL6, CCR1, PDGFD, SGK1, TGFBR3, FCGR1A, FCGR1B, FPR1, and S100A12\}, $\{M I R 223+M A F B\}$ and $\{B S T 1, C 3 A R 1, C D 14$, CLEC4D, CR1, FPR1, FPR2, S100A12, SLC11A1, and TLR2\}.

\section{The validation analysis of differential expression of genes in identified MI transcriptional signatures using GEO datasets}

To confirm the differential expression of genes in identified MI transcriptional signatures we used open datasets GSE62646 and GSE59867 from GEO database, in which gene expression profiles in PBMC of MI patients and healthy individuals without a history of cardiovascular diseases (CVD) were investigated using GeneChip Human Gene 1.0 ST Array. The genes KIR2DL1, KIR2DL3, FCGR1A and FCGR1B that were according to our results included in MI transcriptional signatures were not represented on this array and were therefore excluded from the corresponding MI transcriptional signatures on further consideration. Thus, in a further analysis, 25 genes were considered.

Of the five MI transcriptional signatures we identified, the differential expression of all genes included in the ADAP2-, KLRB1-, and MIR223-containing MI transcriptional signatures was validated in both open datasets GSE62646 and GSE59867 (Table 3). The differential expression of all genes included in the MIR21-containing MI transcriptional signature and MI transcriptional signature from "Neutrophil degranulation" Reactome gene set was validated in GSE59867 dataset. The differential expression of a number of these genes was validated also on GSE62646 dataset with the exception of FPR1 and $S G K 1$ from the MIR21-containing signature and BST1, CLEC4D, FPR1, FPR2 and TLR2 from the "Neutrophil degranulation" signature; these genes were excluded from the corresponding MI transcriptional signatures on further consideration.

Thus, after the validation analyses $\mathrm{MI}$ transcriptional signatures look as follows: $\{A D A P 2\},\{K L R B 1+K L R C 1$, KLRD1, KLRF1\}, \{MIR21+BCL6, CCR1, PDGFD, TGFBR3, S100A12\}, $\{M I R 223+M A F B\}$ and $\{C 3 A R 1$, CD14, CR1, S100A12, SLC11A1\}.

\section{The diagnostic value of the identified MI transcriptional signatures}

The design of our study does not allow to assess the causality between MI and validated transcriptional signatures, which does not exclude the possibility of considering them as diagnostic biomarkers. Their diagnostic value can be assessed by the quality of the classification 
Table 3 The expression of genes from identified MI transcriptional signatures based on our data and data obtained from GSE62646 and GSE59867 GEO datasets

\begin{tabular}{|c|c|c|c|c|c|c|c|}
\hline \multirow[t]{2}{*}{ MI signature } & \multirow[t]{2}{*}{ Gene } & \multicolumn{2}{|c|}{ Our data } & \multicolumn{2}{|c|}{ GSE62646 } & \multicolumn{2}{|c|}{ GSE59867 } \\
\hline & & LOGFC & $P$-value & $\log \mathrm{FC}$ & $P$-value & LogFC & $P$-value \\
\hline ADAP2-containing $\mathrm{Ml}$ signature & ADAP2 & 0.64 & $8.70 E-05$ & 0.62 & $6.40 E-08$ & 0.42 & $4.54 \mathrm{E}-13$ \\
\hline \multirow[t]{4}{*}{ KLRB1-containing $\mathrm{MI}$ signature } & $K L R B 1$ & -0.64 & $3.82 \mathrm{E}-05$ & -0.57 & $1.27 \mathrm{E}-03$ & -0.44 & $2.85 E-06$ \\
\hline & $K L R C 1$ & -0.69 & $1.09 \mathrm{E}-02$ & -0.80 & $3.60 E-05$ & -0.70 & $5.28 \mathrm{E}-10$ \\
\hline & KLRD1 & -0.77 & $3.78 \mathrm{E}-04$ & -0.69 & $1.64 \mathrm{E}-04$ & -0.70 & 7.77E-13 \\
\hline & KLRF1 & -0.65 & $1.90 \mathrm{E}-03$ & -0.79 & $1.06 \mathrm{E}-03$ & -0.70 & $1.20 E-08$ \\
\hline \multirow[t]{8}{*}{ MIR21-containing MI signature } & MIR21 & 1.51 & $5.45 \mathrm{E}-03$ & 0.36 & $3.57 \mathrm{E}-02$ & 0.80 & $2.03 E-12$ \\
\hline & $B C L 6$ & 0.80 & $3.38 \mathrm{E}-03$ & 0.51 & $1.06 \mathrm{E}-04$ & 0.48 & $2.37 E-12$ \\
\hline & CCR1 & 0.55 & $1.97 \mathrm{E}-02$ & 0.57 & $1.63 \mathrm{E}-04$ & 0.67 & $1.29 E-13$ \\
\hline & PDGFD & -0.52 & $1.29 \mathrm{E}-04$ & -0.68 & $1.56 \mathrm{E}-04$ & -0.65 & $3.06 \mathrm{E}-12$ \\
\hline & SGK1 & 0.54 & $3.91 \mathrm{E}-02$ & $\underline{0.18}$ & $\underline{3.00 E-01}$ & 0.34 & $4.78 \mathrm{E}-04$ \\
\hline & TGFBR3 & -0.56 & $7.32 \mathrm{E}-03$ & -0.59 & $1.06 \mathrm{E}-03$ & -0.58 & $4.65 E-11$ \\
\hline & FPR1 & 0.66 & $6.74 \mathrm{E}-03$ & $\underline{0.28}$ & $\underline{5.20 E-02}$ & 0.52 & $7.58 \mathrm{E}-12$ \\
\hline & S100A12 & 0.70 & $4.78 \mathrm{E}-03$ & 0.43 & $4.71 \mathrm{E}-03$ & 0.55 & $1.07 E-10$ \\
\hline \multirow[t]{2}{*}{ MIR223-containing MI signature } & MIR223 & 0.63 & $9.43 E-03$ & 0.61 & $6.85 E-05$ & 0.53 & $4.85 E-09$ \\
\hline & $M A F B$ & 0.65 & $2.16 \mathrm{E}-02$ & 0.45 & $5.70 E-04$ & 0.52 & $7.80 \mathrm{E}-12$ \\
\hline \multirow{10}{*}{ Neutrophil degranulation MI signature } & BST1 & 0.54 & $5.13 \mathrm{E}-03$ & $\underline{0.12}$ & $\underline{2.70 E-01}$ & 0.50 & $9.74 \mathrm{E}-15$ \\
\hline & C3AR1 & 0.60 & $1.88 \mathrm{E}-02$ & 0.34 & $3.14 \mathrm{E}-02$ & 0.48 & $1.07 E-06$ \\
\hline & CD14 & 0.57 & $5.09 \mathrm{E}-03$ & 0.48 & $3.67 \mathrm{E}-05$ & 0.60 & $5.79 E-18$ \\
\hline & CLEC4D & 0.81 & $3.75 E-02$ & $=0.29$ & $\underline{6.48 \mathrm{E}-02}$ & 0.22 & $1.72 E-02$ \\
\hline & $C R 1$ & 0.58 & $3.57 \mathrm{E}-02$ & 0.62 & $9.67 \mathrm{E}-04$ & 0.64 & $1.51 E-13$ \\
\hline & FPR1 & 0.66 & $6.74 \mathrm{E}-03$ & $\underline{0.28}$ & $\underline{5.20 E-02}$ & 0.52 & $7.58 E-12$ \\
\hline & FPR2 & 0.65 & $4.06 \mathrm{E}-02$ & $\underline{0.34}$ & $\underline{6,67 \mathrm{E}-02}$ & 0.63 & $6.28 \mathrm{E}-10$ \\
\hline & S100A12 & 0.70 & $4.78 \mathrm{E}-03$ & 0.43 & $4.71 \mathrm{E}-03$ & 0.55 & $1.07 E-10$ \\
\hline & SLCI1A1 & 0.59 & $2.38 \mathrm{E}-04$ & 0.54 & $6.12 \mathrm{E}-05$ & 0.50 & $2.58 \mathrm{E}-12$ \\
\hline & $T L R 2$ & 0.70 & $5.09 \mathrm{E}-03$ & $\underline{0.06}$ & $5.93 \mathrm{E}-01$ & 0.22 & $3.57 E-04$ \\
\hline
\end{tabular}

Underline indicates $p$ value $>0.05$

of MI patients from healthy controls. To search for such an optimal classifier, a L2 regularized logistic regression model was trained on the GSE59867 dataset (Fig. 4).

Figure 4a shows that MI patients at the time of admission to hospital could be classified from healthy individuals based on the selected MI transcriptional signatures, and the quality of the classification model on the test dataset ( $A U C=0.926)$ is slightly higher than on the training dataset $(\mathrm{AUC}=0.910)$, illustrating the stability of the model and the lack of its overfitting. While analyzing the available data from GSE62646 dataset on the levels of gene expression during the 6-month follow-up after MI (Fig. 4b), we observed that the classification model remains effective within 6 days after MI onset (AUC $=0.773$, blue line) but 6 month after MI onset the effectiveness of this model considerably decreases (AUC $=0.594$, red line).

For the feature selection and to reduce the number of DEGs included in the classification model we used a logistic regression with the L1-norm penalty function
(Fig. 5). As a result, $A D A P 2, K L R C 1, M I R 21, P D G F D$ and $C D 14$ genes were selected for the classification model as the most important DEGs (Fig. 5a). ROC-curves constructed for these genes are demonstrated in Figs. 5b, c. The comparison of ROC-curves from Figs. 4 and 5 demonstrates that the quality of the classification on test dataset slightly decreased from 0.926 (dark green curve on Fig. 4b) to 0.911 after applying L1 regularization (dark green curve on Fig. 5b). While analyzing the changes in the quality of the classification model based on the levels of gene expression over time after MI onset, ROC-AUC values also slightly changed after applying L1 regularization (see Figs. 4b and 5c). Thus, five DEGs are sufficient for the classification; among these genes DEGs from MIR223-containing MI transcriptional signature were not presented. 

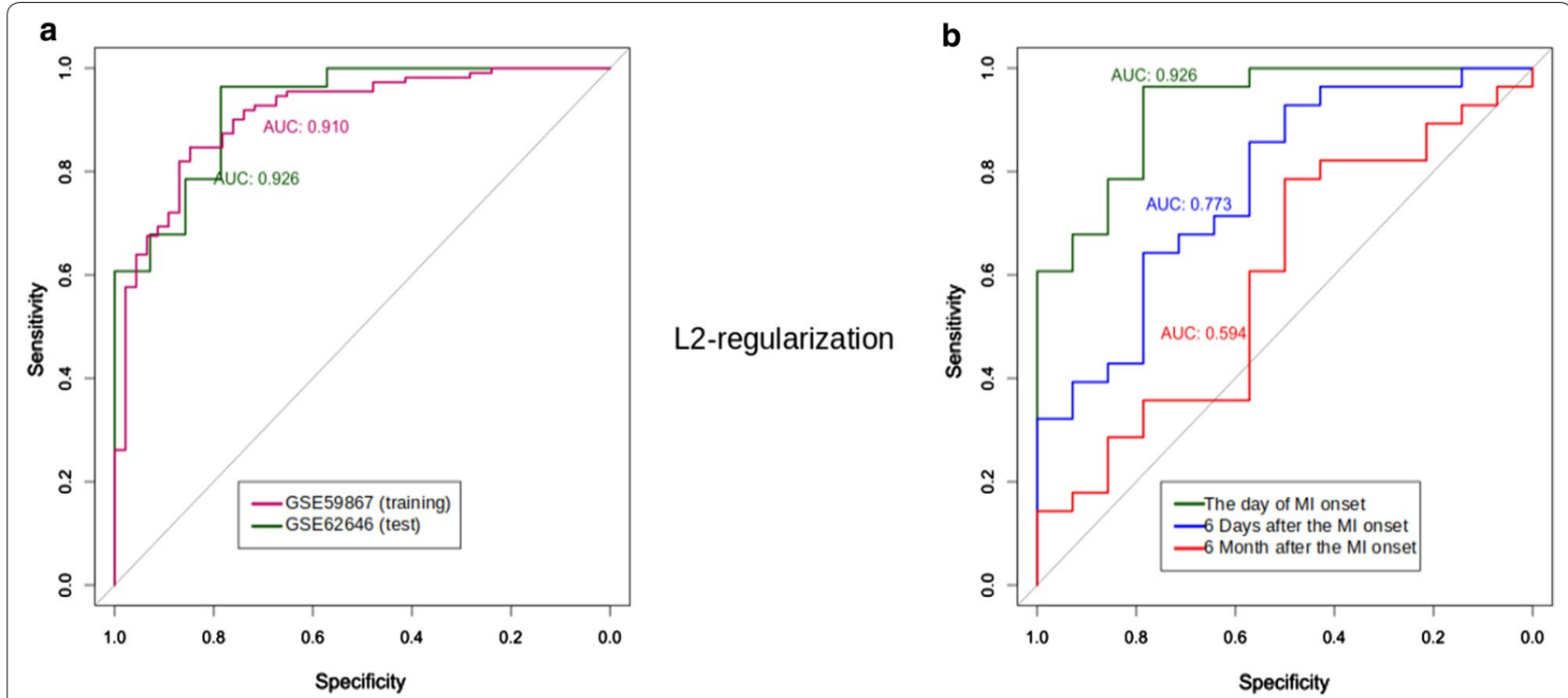

Fig. 4 Quality and robustness of the classification model with a L2-norm penalty function based on the cumulative expression levels of genes included in considered MI transcriptional signatures: $\{A D A P 2\},\{K L R B 1+K L R C 1, K L R D 1, K L R F 1\},\{M I R 21+B C L 6, C C R 1, P D G F D, T G F B R 3, S 100 A 12\}$, $\{$ MIR223 + MAFB\} and $\{C 3 A R 1, C D 14, C R 1$, S100A12, SLC11A1\}. a Areas Under receiver operating characteristic Curve (ROC-AUC) for the training (GSE59867) and test (GSE62646) datasets. b Time-depended (starting from MI onset) ROC-AUC metrics of the classification model

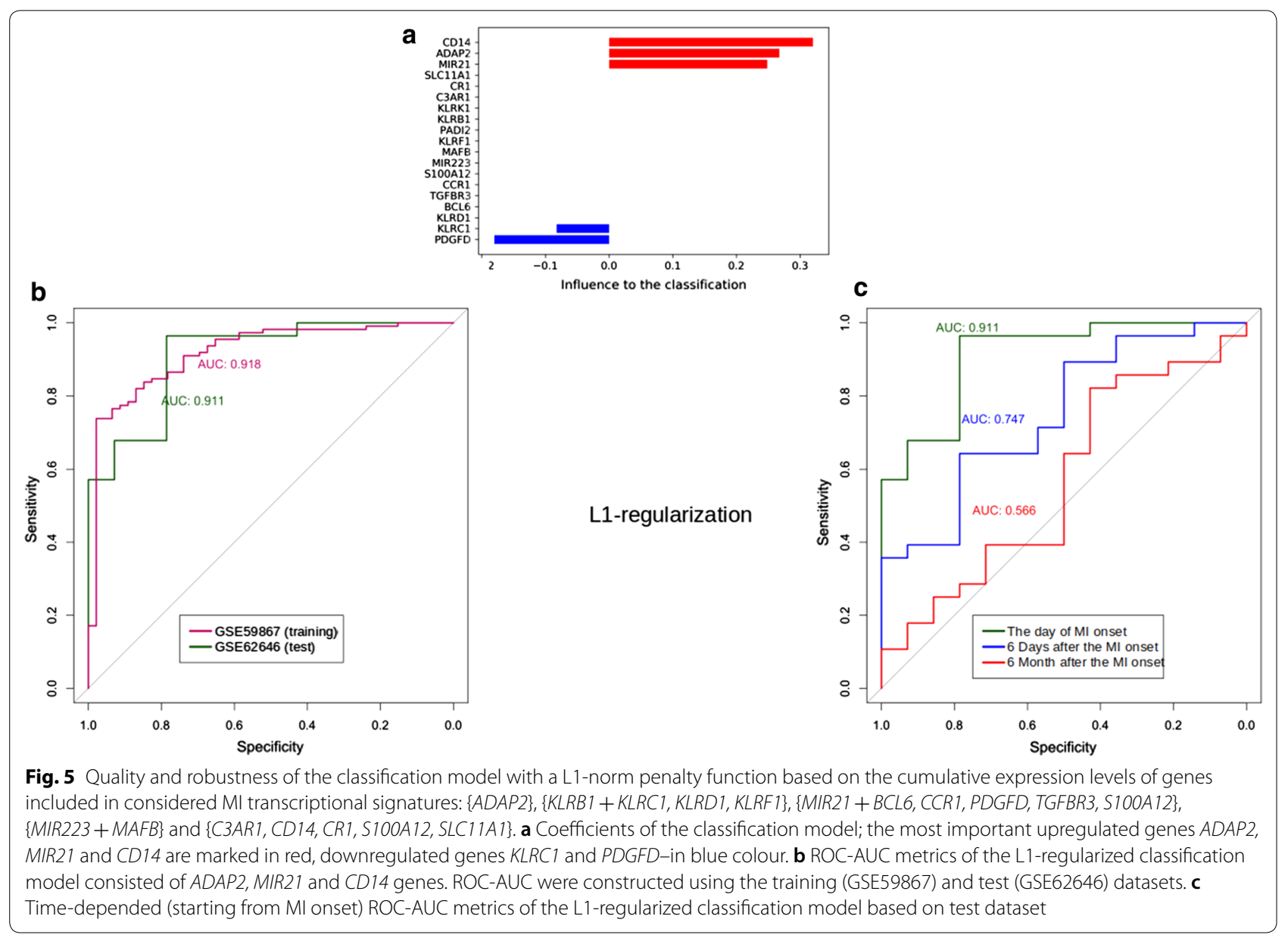




\section{Discussion}

In the study we searched for MI transcriptional signatures (individual dysregulated genes or functional patterns of dysregulated genes) that could be potentially used in MI diagnosis. We compared the transcriptome profiles in PBMC of patients with first MI and healthy individuals using GeneChip Human Transcriptome Array 2.0 and identified five MI transcriptional signatures containing from 1 to 6 DEGs: $\{A D A P 2\},\{K L R B 1+K L R C 1, K L R D 1$, KLRF1\}, \{MIR21+BCL6, CCR1, PDGFD, TGFBR3, S100A12\}, $\{M I R 223+M A F B\}$ and $\{C 3 A R 1, C D 14, C R 1$, S100A12, SLC11A1\}.

In order to select the most important for classification DEGs, further research steps included: validation on open datasets GSE62646 [23] and GSE59867 [24], construction of L2 regularized logistic regression model for estimation the diagnostic value of the MI transcriptional signatures and feature selection using L1-norm penalty function. This approach allowed to exclude from the classification model MI transcriptional signature $(M I R 223+M A F B)$ as insufficiently effective and to reduce the number of DEGs from other signatures to ADAP2, KLRC1, MIR21, PDGFD and CD14. According to the ROC-AUC analysis the obtained classification model, including 5 genes, is enable classifying MI patients and healthy controls with a quality of 0.911 while the quality of initial classification model, including 18 genes, was equal to 0.926 . Thus, a decrease in the number of genes did not significantly affect the quality of the model. A comparable decline in the quality of both classification models over time from MI onset was shown. This decline occurs rather slowly, for days and weeks. After 6 months the cumulative expression levels of genes included in MI signatures almost completely return to the normal levels, which indicates the dependence of their expression on time since the onset of MI as an acute condition.

Patients with obstructive and non-obstructive MI were included in the initial group equally, while there is no information on this characteristic for the patients from datasets GSE62646 [23] and GSE59867 [24] we used for validation. Therefore, there is reason to argue that the transcriptional signatures we identified are associated with MI development regardless of specificities of its pathogenesis. Therefore, there is reason to argue that the transcriptional signatures we identified reflect the development of MI regardless of specificities of its pathogenesis.

Consider consistently the characteristics of genesclassifiers. The gene ADAP2 encodes ArfGAP With Dual $\mathrm{PH}$ Domains 2 protein and was designated in our study as individual MI transcriptional signature; no data on the involvement of this gene in the development of CVD and/or its complications were found. However, the product of this gene was shown to be involved in heart development, and its dysfunction presumably is associated with cardiovascular malformations in NF1 microdeletion syndrome [25].

The gene KLRC1 refers to a MI transcriptional signature containing the genes of killer cell lectin-like receptors (KLR) that encode a family of transmembrane proteins, characterized by a type II membrane orientation and the presence of a C-type lectin domain; they are predominantly expressed in NK cells. The association of some genes from this signature (KLRD1 and KLRC1) with MI or its complications was previously shown by Maciejak et al. and Kiliszek et al., whose data were used for validation analysis in our study [23, 24]. We have shown that the expression of the genes KLRB1, KLRC1, $K L R D 1, K L R F 1$ is consistently decreased in MI, that is in a good accordance with the study by Yan et al. [26], where a loss of NK cell activity was found in patients with acute MI, in particular, due to a decrease in KLRB1 expression.

The MIR21 gene and target genes of miR-21 were included in one MI transcriptional signature, composed mainly of genes from "Cytokine Signaling in Immune system" pathway. Thus, in addition to cytokine signaling pathways, which role in MI development was previously described [27], we have identified and validated on independent GEO datasets the influence of miR-21 through the regulation of this pathway in PBMC during MI. The functional role of miR-21 in cardiac tissue has been studied for a long time, and by now a large amount of data has been accumulated on this subject [28], while in PBMC its role remains unclear. In one of the studies the negative correlation of miR-21 expression level in MI with the levels of IL-1 $\beta$, IL-6, and TNF- $\alpha$ cytokines was shown due to regulatory effect of this miRNA on the expression of KBTBD7; this gene encodes a member of BTB-kelch proteins, kelch repeat and BTB (POZ) domain containing 7 , which promotes inflammatory responses in macrophages [29]. In turn, in our study, the association of miR-21 and its target genes PDGFD, TGFBR3, CCR1 and BCL6 expression levels with MI was demonstrated, from which PDGFD gene encoded platelet derived growth factor D was found to be the most important based on the results of L1 regularization. The genes of the PDGF family and their involvement in the pathogenesis of various diseases are well studied; in particular, PDGFD is known to be involved in the fibrosis and neovascularization of the cardiac tissue [30].

The gene $C D 14$ encodes a receptor on the surface of myeloid cells, which participates in CD14/TLR4/MD2 signaling pathway involved in the recognition of lipopolysaccharides [31]. This gene was identified in our study 
as a component of "Neutrophil degranulation" pathway. The neutrophils are known to be actively involved in the development and elimination of MI consequences [32]. Furthermore, polymorphic variants in $C D 14$ gene were found to be associated with MI [33].

Further investigations are implicitly needed to clarify the causality between MI and the identified MI-associated signatures.

The data on differential expression of a number of genes in PBMC of MI patients obtained in our study were validated on two independent datasets that indicates their value. The identified DEGs could be suitable for the prediction of the first MI before the appearance of the disease symptoms, as it was previously described for some miRNAs [34]. Further investigations are implicitly needed to clarify the functional role of the identified MI-associated genes in the development of this disease.

\section{Conclusions}

The present study implements the pipeline designed to the collapsing the list of differentially expressed in MI genes into a diagnostic signature; the obtained robust classification model includes ADAP2, KLRC1, MIR21, $P D G F D$ and $C D 14$ genes and is enable classifying MI patients and healthy controls with a quality of 0.911 on an independent test data. This confirm the relevanceof our results demonstrating the associations of these genes with MI, which were obtained on small sample set by our pipeline. This pipeline could be useful in high-throughput data analysis for the searching of diagnostic signature of other diseases.

\section{Supplementary information}

Supplementary information accompanies this paper at https://doi. org/10.1186/s12967-020-02400-1.

\footnotetext{
Additional file 1: Table S1. The differentially expressed genes identified in PBMC of MI patients when compared to healthy individuals. Figure S1. Correlation matrix plot of the expression levels of gene MIR21 and target genes of this miRNA. Figure S2. Correlation matrix plot of the expression levels of gene MIR223 and target gene MAFB of this miRNA. Figure S3. Correlation matrix plot of the expression levels of differentially expressed genes from "Neutrophil degranulation" Reactome set. Figure S4. Correlation matrix plot of the expression levels of differentially expressed genes from "Cytokine Signaling in Immune system" Reactome set and of MIR21 and target genes of this miRNA. Figure S5. Correlation matrix plot of the expression levels of differentially expressed genes from "Immunoregulatory interactions between a Lymphoid and a non-Lymphoid cell" Reactome set.
}

\section{Abbreviations}

Ml: Myocardial infarction; CAD: Coronary artery disease; PBMC: Peripheral blood mononuclear cells; DEGs: Differentially expressed genes; CTRLs: Individuals in the control group; CVD: Cardiovascular diseases; CV: Cross-validation; FDR: False discovery rate; NK: Natural killers; KLR: Killer cell lectin-like receptors.

\section{Acknowledgments}

We thank Dr. Roman Shakhnovich, Dr. Nino Kulava and PhD Natalia Matveeva from National Medical Research Center for Cardiology, Russia, for their assistance with MI patients and controls collection. We appreciate PhD Alexander Favorov for valuable advices while producing the manuscript.

\section{Authors' contributions}

Conceptualization, GO, NB and OF: Data curation, GO and PK; Formal analysis, GO; Investigation G.O, NB and PK; Methodology, NB, GO; Project administration, OF; Validation, GO; Visualization, GO; Writing-original draft, GO and OF; Writing-review and editing, GO, NB and OF. All authors read and approved the final manuscript.

\section{Funding}

This study was partially supported by the Grant No.16-14-10251 from the Russian Science Foundation and the Grant No. 075-15-2019-1789 from the Ministry of Science and Higher Education of the Russian Federation allocated to the Center for Precision Genome Editing and Genetic Technologies for Biomedicine.

\section{Availability of data and materials}

All expression data are deposited in the Gene Expression Omnibus international public repository under accession identification as GSE141512 [15].

\section{Ethics approval and consent to participate}

This study was approved by the Ethical Committee of the Pirogov Russian National Research Medical University, Moscow and informed consent was obtained from all patients.

\section{Consent for publication}

Not applicable.

\section{Competing interests}

The authors declare that they have no conflict of interest relating to the conduct of this study or the publication of this manuscript.

\section{Author details}

${ }^{1}$ National Medical Research Center for Cardiology, Moscow 121552, Russia.

2 Pirogov Russian National Research Medical University, Moscow 117997, Russia.

Received: 26 February 2020 Accepted: 3 June 2020

Published online: 09 June 2020

References

1. Roberts R. Genetics of coronary artery disease. Circ Res. 2014;114:1890903. https://doi.org/10.1161/CIRCRESAHA.114.302692.

2. MY. Wu, CJ. Li, MF. Hou, PY. Chu. New Insights into the role of inflammation in the pathogenesis of atherosclerosis, Int. J. Mol. Sci. 18 (2017) pii: E2034.

3. Ramji DP, Davies TS. Cytokines in atherosclerosis: Key players in all stages of disease and promising therapeutic targets. Cytokine Growth Factor Rev. 2015;26:673-85. https://doi.org/10.1016/j.cytogfr.2015.04.003.

4. Ross R. Atherosclerosis-An inflammatory disease. N Engl J Med. 1999:340:115-26. https://doi.org/10.1056/NEJM199901143400207.

5. Frangogiannis NG. The inflammatory response in myocardial injury, repair, and remodelling. Nat Rev Cardiol. 2014;11:255-65.

6. Jung M, Dodsworth M, Thum T. Inflammatory cells and their non-coding RNAs as targets for treating myocardial infarction. Basic Res Cardiol. 2019;114(1):4

7. Zawada AM, Rogacev KS, Müller S, Rotter B, Winter P, Fliser D, Heine GH. Massive analysis of CDNA Ends (MACE) and miRNA expression profiling identifies proatherogenic pathways in chronic kidney disease. Epigenetics. 2014;9:161-72.

8. N. Peláez, R.W. Carthew, Biological robustness and the role of microRNAs: a network perspective, in: Curr. Top. Dev. Biol., Elsevier, 2012: pp. 237-255.

9. Barabási A-L, Gulbahce N, Loscalzo J. Network medicine: a network-based approach to human disease. Nat Rev Genet. 2011;12:56-68. https://doi. org/10.1038/nrg2918. 
10. Lai $X$, Wolkenhauer $O$, Vera J. Understanding microRNA-mediated gene regulatory networks through mathematical modelling. Nucleic Acids Res. 2016;44:6019-35.

11. de Couto G, Gallet R, Cambier L, Jaghatspanyan E, Makkar N, Dawkins JF, Berman BP, Marbán E. Exosomal microRNA transfer into macrophages mediates cellular postconditioning. Circulation. 2017;136:200-14.

12. Cheng M, An S, Li J. Identifying key genes associated with acute myocardial infarction. Medicine. 2017;96:e7741.

13. Thygesen K, Alpert JS, Jaffe AS, Simoons ML, Chaitman BR, White HD. Third universal definition of myocardial infarction. Circulation. 2012;126:2020-35.

14. Gautier L, Cope L, Bolstad BM, Irizarry RA. affy-analysis of Affymetrix GeneChip data at the probe level. Bioinformatics. 2004;20:307-15.

15. Durinck S, Moreau Y, Kasprzyk A, Davis S, De Moor B, Brazma A, Huber W. BioMart and Bioconductor: a powerful link between biological databases and microarray data analysis. Bioinformatics. 2005;21:3439-40.

16. GK. Smyth, Limma: linear models for microarray data, in: Bioinforma. Comput. Biol. Solut. Using R Bioconductor, Springer, 2005: pp. 397-420.

17. GEO DataSet, (n.d.). https://www.ncbi.nlm.nih.gov/geo/query/acc. cgi?acc $=$ GSE141512

18. Chou C-H, Shrestha S, Yang C-D, Chang N-W, Lin Y-L, Liao K-W, Huang W-C, Sun T-H, Tu S-J, Lee W-H. miRTarBase update, a resource for experimentally validated microRNA-target interactions. Nucleic Acids Res. 2018;46(2017):D296-302.

19. Fabregat A, Jupe S, Matthews L, Sidiropoulos K, Gillespie M, Garapati P, Haw R, Jassal B, Korninger F, May B, Milacic M, Roca CD, Rothfels K, Sevilla C, Shamovsky V, Shorser S, Varusai T, Viteri G, Weiser J, Wu G, Stein L, Hermjakob H, D'Eustachio P. The reactome pathway knowledgebase. Nucleic Acids Res. 2018;46:D649-55. https://doi.org/10.1093/nar/gkx1132.

20. A. Hagberg, P. Swart, DS Chult, Exploring network structure, dynamics, and function using NetworkX, in: Pasadena. USA., 2008: p. 11.

21. Szklarczyk D, Morris JH, Cook H, Kuhn M, Wyder S, Simonovic M, Santos A, Doncheva NT, Roth A, Bork P, Jensen LJ, von Mering C, The STRING. The STRING database in 2017: quality-controlled protein-protein association networks, made broadly accessible. Nucleic Acids Res. 2017;45(2017):D362-8. https://doi.org/10.1093/nar/gkw937.

22. Pedregosa F, Varoquaux G, Gramfort A, Michel V, Thirion B, Grisel O, Blondel M, Prettenhofer P, Weiss R, Dubourg V. Scikit-learn: machine learning in Python. J Mach Learn Res. 2011;12:2825-30.

23. Kiliszek M, Burzynska B, Michalak M, Gora M, Winkler A, Maciejak A, Leszczynska A, Gajda E, Kochanowski J, Opolski G. Altered gene expression pattern in peripheral blood mononuclear cells in patients with acute myocardial infarction. PLOS ONE. 2012;7:e50054.

24. Maciejak A, Kiliszek M, Michalak M, Tulacz D, Opolski G, Matlak K, Dobrzycki S, Segiet A, Gora M, Burzynska B. Gene expression profiling reveals potential prognostic biomarkers associated with the progression of heart failure. Genome Med. 2015;7:26.

25. Venturin M, Carra S, Gaudenzi G, Brunelli S, Gallo GR, Moncini S, Cotelli F, Riva P. ADAP2 in heart development: a candidate gene for the occurrence of cardiovascular malformations in NF1 microdeletion syndrome. J Med Genet. 2014;51:436-43.

26. Yan W, Zhou L, Wen S, Duan Q, Huang F, Tang Y, Liu X, Chai Y, Wang L. Differential loss of natural killer cell activity in patients with acute myocardial infarction and stable angina pectoris. Int J Clin Exp Pathol. 2015;8:14667.

27. Fang L, Moore XL, Dart AM, Wang LM. Systemic inflammatory response following acute myocardial infarction. J Geriatr Cardiol. 2015;2015(12):305-12. https://doi.org/10.11909/j.i ssn.1671-5411.2015.03.020.

28. CK Sen, S. Roy, MicroRNA 21 in tissue injury and inflammation: Authors' Retrospective, Oxford University Press, 2012.

29. Yang L, Wang B, Zhou Q, Wang Y, Liu X, Liu Z, Zhan Z. MicroRNA-21 prevents excessive inflammation and cardiac dysfunction after myocardial infarction through targeting KBTBD7. Cell Death Dis. 2018;9:769.

30. Folestad E, Kunath A, Wågsäter D. PDGF-C and PDGF-D signaling in vascular diseases and animal models. Mol Aspects Med. 2018;1 (62):1.

31. Kitchens RL. Role of CD14 in cellular recognition of bacterial lipopolysaccharides. Chem Immunol. 2000;74:61-82.

32. Lai S-L, Marín-Juez R, Stainier DYR. Immune responses in cardiac repair and regeneration: a comparative point of view. Cell Mol Life Sci. 2019;76:1365-80. https://doi.org/10.1007/s00018-018-2995-5.

33. Kondo T, Ohno M, Shimokata K, lino S, Inden Y, Murohara T, Hirai M. CD14 promoter polymorphism is associated with acute myocardial infarction resulting from insignificant coronary artery stenosis. Heart. 2003;89:931-2

34. Bye A, Røsjø H, Nauman J, Silva GJ, Follestad T, Omland T, Wisløff U. Circulating microRNAs predict future fatal myocardial infarction in healthy individuals-the HUNT study. J Mol Cell Cardiol. 2016;97:162-8.

\section{Publisher's Note}

Springer Nature remains neutral with regard to jurisdictional claims in published maps and institutional affiliations.
Ready to submit your research? Choose BMC and benefit from:

- fast, convenient online submission

- thorough peer review by experienced researchers in your field

- rapid publication on acceptance

- support for research data, including large and complex data types

- gold Open Access which fosters wider collaboration and increased citations

- maximum visibility for your research: over $100 \mathrm{M}$ website views per year

At BMC, research is always in progress.

Learn more biomedcentral.com/submissions 